Paper presented at the Particle Accelerator Conference, Portland, OR, May 12-16, 2003.

BNL-71.402-2003-CP

\title{
INITIAL TEST OF A FAST-RAMPED SUPERCONDUCTING MODEL DIPOLE FOR GSI'S PROPOSED SIS200 ACCELERATOR *
}

\author{
P. Wanderer ${ }^{\dagger}$, M. Anerella, G. Ganetis, A. Ghosh, P. Joshi, A. Marone, J. Muratore, J. Schmalzle, \\ R. Soika, R. Thomas BNL, Upton, NY 11973-5000, USA \\ J. Kaugerts, G. Moritz, GSI, Darmstadt, Germany \\ W. Hassenzahl, Advanced Energy Analysis, Piedmont, CA 94611 USA \\ M. N. Wilson, consultant, 33 Lower Radley, Abingdon OX14 3AY, UK
}

\section{Abstract}

Gesellschaft fur Schwerionenforschung (GSI) has proposed a large expansion of the existing facility in Darmstadt, Germany. The proposal includes an accelerator, SIS200, with rigidity of $200 \mathrm{~T} \cdot \mathrm{m}$ that utilizes $4 \mathrm{~T}$ superconducting dipoles ramped at $1 \mathrm{~T} / \mathrm{s}$. An R\&D program including both the superconductor and the magnet is directed at achieving the desired ramp rate with minimal energy loss. The RHIC arc dipoles, with $8 \mathrm{~cm}$ aperture, possess adequate aperture and field strength but are ramped at only $1 / 20$ of the desired rate. However, for reasons of speed and economy, the RHIC dipole is being used as the basis for this work. The superconductor $R \& D$ has progressed far enough to permit the manufacture of an initial cable with satisfactory properties. This cable has been used in the construction of a $1 \mathrm{~m}$ model magnet, appropriately modified from the RHIC design. The magnet has been tested successfully at $2 \mathrm{~T} / \mathrm{s}$ to $4.38 \mathrm{~T}$.

\section{INTRODUCTION}

This paper reports the successful initial test of a $1 \mathrm{~m}$ fast-ramped superconducting model dipole built as part of the magnet $R \& D$ program for the new accelerator facility that will be built at the Gesellschaft fur Schwerionenforschung (GSI) in Darmstadt, Germany [1]. The magnet was designed to meet specifications for the SIS200 accelerator: $4 \mathrm{~T}$ central field, $1 \mathrm{~T} / \mathrm{sec}$ ramp rate $[2,3]$. Because the RHIC arc dipoles [4] can operate at 4 T, GSI and BNL are working together on a model magnet program based on the RHIC design. Use of the RHIC cross-section has enabled the work to take advantage of much of the RHIC design and tooling and some of the RHIC magnet components, thereby getting the effort off to a fast start. However, it has been necessary to make significant modifications to the magnet design, especially the superconductor, to build a magnet that can ramp 20 times faster than RHIC and have much lower eddy current energy losses.

*Work supported by US DOE under contract DE-AC02-98CH10886 and GSI.

† wanderer@bnl.gov

\section{SUPERCONDUCTOR}

The strand for the first dipole (GSI001) is made fiom leftover $\mathrm{NbTi}$ strand from the RHIC program (2.25 Cu:SC ratio, $\mathrm{Jc}=2900 \mathrm{~A} / \mathrm{mm}^{2}$ at $5 \mathrm{~T}, 4.2 \mathrm{~K}, 0.648 \mathrm{~mm}$. wire diameter, $6 \mu \mathrm{m}$ filament diameter, $13 \mu \mathrm{mm}$ twist pitch). The twist-pitch of the GSI001 strand is $4 \mathrm{~mm}$. The extra twist reduced the wire diameter slightly $(\sim 13 \mu \mathrm{m})$ with minimal impact $(<5 \%)$ on the critical current. The cable parameters that determine the energy loss are the resistance between adjacent strands, $R_{a}$, and the resistance between crossing strands, $R_{c}$ [5]. In the cable used in this magnet (GSI\#004), $R_{c}$ was increased compared to RHIC values by inserting two $25 \mu \mathrm{m}$-thick, $8 \mathrm{~mm}$ wide stainless steel cores into the cable as it was formed. To allow for some current sharing, $R_{a}$ was decreased by coating the strands with Staybrite ( $\mathrm{Sn}$ with $4 \% \mathrm{Ag}$ ). Without cores, cables made from Staybrite coated strands (e.g., LHC) have $R_{c} \sim 20 \mu \Omega$. The cables used in this magnet have $R_{c}$ $=60 \mathrm{~m} \Omega$, more than a factor of a thousand higher. The measured value of $R_{a}$, is much lower, $64 \mu \Omega$, so eddy current effects are dominated by currents flowing between adjacent wires. (This value of $R_{a}$ was determined using the standard method. Detailed measurements reveal that $R_{a}$ actually varies by as much as a factor of $\sim 1000$ between the wide and narrow sections of the cable. The effects of this variation introduce some uncertainty into the calculation of energy loss [6].)

The cable has standard RHIC insulation: two wraps of Kapton(B), each $25 \mu \mathrm{m}$ thick, with $50 \%$ overlap of each wrap, and a polyimide-based heat-set adhesive on the outside of the inner wrap and on both sides of the outer wrap. This provides substantial impedance to the flow of helium between the interior of the cable and the reservoir just outside the coil. To allow more rapid heat exchange, a laser was used to cut away about $25 \%$ of the insulation on the thin edge of the insulated cable (Fig. 1). The holes in the insulation were precisely made so that the coils could be wound and cured without developing turn-toturn shorts. Turn-to-turn standoff voltages of $1.1 \mathrm{kV}$ were observed for both the straight section and end regions of a test coil that was cut in half and collared. This is less than the nominal test condition, $>2 \mathrm{kV}$, for RHIC coils but sufficient for the SIS200 application. 


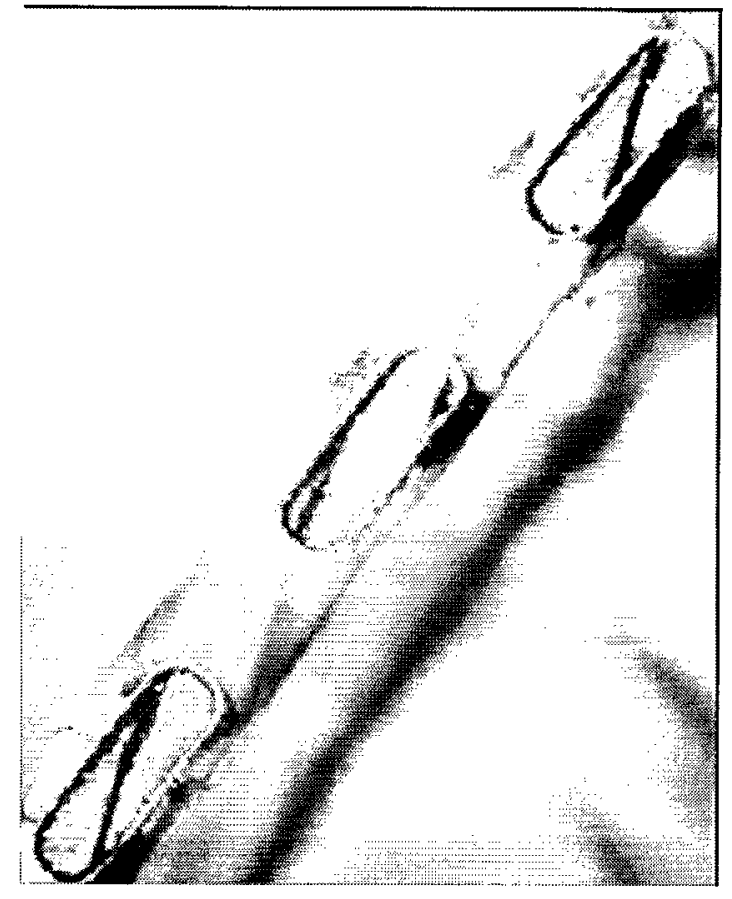

Figure 1. Cable inner edge.

\section{MAGNET CONSTRUCTION}

Where feasible, magnet components were made from insulators rather than metals. In the GSI magnet (Fig. 2), the three wedges used in the coil to control field quality were G11 rather than $\mathrm{Cu}$. The cable is $\sim 25 \mu \mathrm{m}$ thicker than the RHIC cable, resulting in a cured coil (32 turns) that is oversize by $0.9 \mathrm{~mm}$. The G10 end pole spacers and Ultem $(\mathbb{B})$ coil end saddles were modified to take account of the oversize cable. The shims placed between the coil and the pole were made of G11 and reduced in thickness to compensate for the oversized coil.

The coils were collared with Kawasaki high-Mn stainless steel collars. (The collars were designed for the LHC D2/D4 IR dipoles, also made using a variation of the RHIC arc dipole design [7].) As a handling aid, collars are assembled into $15 \mathrm{~cm}$-long packs before being placed on the coils. G10 tubes were used to assemble these packs. It was possible to replace the brass keys used to lock the collars around the coils with G11 everywhere except for $2 \mathrm{~cm}$ at the nonlead end, where brass was used. At the lead end of the magnet, the collars have a larger inner diameter because of the radial space needed to bring the lead at the pole of the coil beyond the end of the coil. For the GSI magnet, the brass pieces used to fill this volume were halved in thickness and doubled in quantity, and the pieces of brass were insulated from one another.

The yoke laminations were $0.5 \mathrm{~mm}$ thick and punched from low coercivity steel $\left(\mathrm{H}_{\mathrm{c}}=31 \mathrm{~A} / \mathrm{m}\right)$ with $3.3 \% \mathrm{Si}$ added. The laminations were coated with B-stage epoxy and glued into blocks $254 \mathrm{~mm}$ long. Five blocks make up a half yoke. Each half yoke is supported against axial motion by three stainless steel rods that run through holes
GSI COLDMASS CROSS SECTION

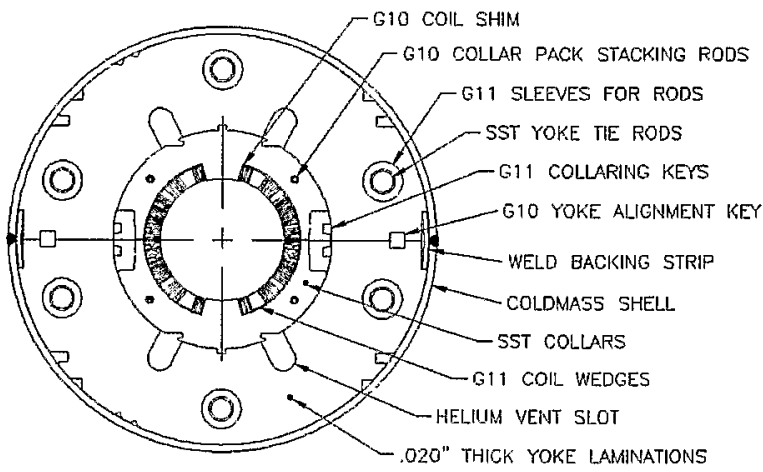

Figure 2. Cross section of the cold mass.

in the yoke and restrain the yoke with stainless steel nuts insulated from the yoke by G10 washers. G10 tubes are placed around the stainless steel rods to insulate the rods from the yoke. The two yoke halves are aligned with respect to one another with G10 alignment keys at the yoke midplane. The yoke is held together by welding a stainless steel shell around it. There is a welding backup strip at the midplane but the strip is not welded to the yoke.

In RHIC magnets, quenches at high ramp rate are likely to originate in the "ramp" section of the cable, at the end of the pole turn of the coil, where the cable is filled with solder to keep it rigid as it is moved by G10 fixtures to a larger radius so that it can be brought past the end of the coil and spliced to the cable from the other coil. The splice between the two coils halves is itself a possible source of quenching. However, the high value of $R_{c}$ in the cored cable was judged to be sufficient to prevent such quenching in this magnet, so this region of magnet construction was the same as for RHIC dipoles.

The magnet was constructed so that helium axial flow would be forced along the coil inner radius, for maximum cooling. All other axial flow paths were blocked either by plugs or vents. Vents (reed valves), located at each end of the yoke, will open under pressure to allow helium to escape during a quench. (These features will be tested when the magnet is tested at GSI in supercritical helium. BNL testing is in pool boiling.) The magnet has no beam tube in it. Standard RHIC construction is used to restrain the axial motion of the coil.

\section{QUENCH TEST RESULTS}

The magnet was tested in pool boiling helium $(4.5 \mathrm{~K}$ nominal). It was initially operated at the RHIC ramp rate, $0.053 \mathrm{~T} / \mathrm{s}$ (Fig. 3). The sixth and last quench at this ramp rate was at $7.76 \mathrm{kA}$ (4.38 T central field), approximately equal to the short-sample limit of the cable and $\sim 10 \%$ above the $4 \mathrm{~T}$ design. A different power supply was used for quenching at high ramp rate. It was found that both the magnet and this supply could ramp at $2 \mathrm{~T} / \mathrm{s}$, twice the design. Quench testing was carried out at this ramp rate because it reduced the DC heating of the power supply 


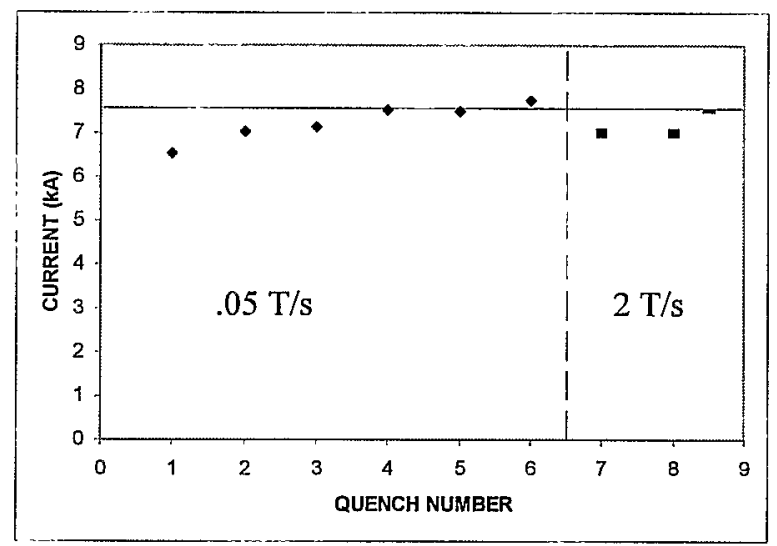

Figure 3. Quench history, showing six quenches at $0.05 \mathrm{~T} / \mathrm{s}$, followed by two at $2 \mathrm{~T} / \mathrm{s}$, then stable operation at $7.5 \mathrm{kA}$. The horizontal line indicates the estimated short-sample limit of the magnet.

leads, one of the power supply operational limits. The initial part of the program to measure energy losses was to cycle the magnet to $7000 \mathrm{~A}$. It quenched a few moments after reaching $7000 \mathrm{~A}$ the first two times it was ramped to this current. Subsequently it was ramped numerous times to currents as high as $7500 \mathrm{~A}$ (the limit of the supply) at 2 $\mathrm{T} / \mathrm{sec}$ without quenching. Thus, the magnet has substantially exceeded the quench current and ramp rate goals of the GSI design.

\section{FUTURE PLANS}

Work on the energy loss measurement is still underway and will be published at a later time.

Since this work was initiated, the energy of the accelerator has been increased $50 \%$. The design value of the dipole central field has been increased, to $6 \mathrm{~T}$, a value beyond that which can be readily achieved in a magnet built with a single layer coil. Consequently, future plans for BNL/GSI work now focus on establishing design principles for such magnets.

The highest priority is the measurement of the magnetic fields during the $1 \mathrm{~T} / \mathrm{s}$ ramp. Development of the new type of measurement system needed for this is underway. The present magnet will be tested in supercritical helium at GSI. The addition of ventilation holes to the inner edge of the cable is expensive, and at least one magnet should be built and tested to determine whether this venting is necessary. The use of two cores in the cable became necessary when holes in cable made with a single core were found in the core near the keystoned cable's narrow edge at the places where the wires crossed. Making cable with two cores is more difficult that making them with one core, so some further development was undertaken, resulting in the successful production of cable with a single core but no holes. To reduce losses further, strand with $3.5 \mathrm{~mm}$ filaments has been ordered.

\section{CONCLUSIONS}

A model magnet for the GSI SIS200 superconducting accelerator has been rapidly built using many components from the BNL RHIC arc dipoles. It has been successfully ramped at $2 \mathrm{~T} / \mathrm{s}$, forty times higher than the RHIC ramp rate and twice the design goal, to $4.38 \mathrm{~T}$, above the $4 \mathrm{~T}$ design field.

\section{REFERENCES}

[1] Gesellschaft Fur Schwerionenforschung, "An International Accelerator Facility for Beams of Ions and Antiprotons," Conceptual Design Report, November 2001.

[2] G. Moritz et al, "Towards Fast-Pulsed Superconducting Synchrotron Magnets," Proc. 2001 Particle Accelerator Conf. (Chicago IL, June 18-22, 2001), p. 211.

[3] G. Moritz, "Superconducting Magnets for the "International Accelerator Facility for Beams of Ions and Anti-protons' at GSI," Proc. 2002 Applied Superconductivity Conf., (Houston TX, Aug. 4-9, 2002).

[4] M. Anerella et al., "The RHIC Magnet System," Nucl. Instrum. Meth. A 499 (2003) pp. 280-315.

[5] M. N. Wilson et al, "Design Studies on Superconducting $\cos \theta$ Magnets for a Fast Pulsed Synchrotron," IEEE Trans. Appl. Superconductivity, Vol. 12, No. 1 (March 2002), p. 313 [MT17].

[6] M. N. Wilson et al, "Cored Rutherford Cables for the GSI Fast Ramping Synchrotron," Proc. Proc. 2002 Applied Superconductivity Conf., (Houston TX, Aug. 4-9, 2002).

[7] J. Muratore et al., "Test Results for Initial Production of LHC Insertion Region Dipole Magnets," Proc. 2002 European Particle Accelerator Conf. (Paris, June 3-7, 2002), p. 2415. 